\title{
Four New Species and Additional Distributions of Elaphoglossum Section Elaphoglossum (Dryopteridaceae) from the Neotropics
}

\author{
Alexander Francisco Rojas-Alvarado \\ Universidad Nacional de Costa Rica, Heredia, Costa Rica \\ Email: alfrojasa@yahoo.com
}

How to cite this paper: Rojas-Alvarado, A.F. (2017) Four New Species and Additional Distributions of Elaphoglossum Section Elaphoglossum (Dryopteridaceae) from the Neotropics. American Journal of Plant Sciences, 8, 1234-1247.

https://doi.org/10.4236/ajps.2017.86082

Received: April 6, 2017

Accepted: May 16, 2017

Published: May 19, 2017

Copyright $\odot 2017$ by author and Scientific Research Publishing Inc. This work is licensed under the Creative Commons Attribution International License (CC BY 4.0).

http://creativecommons.org/licenses/by/4.0/

\begin{abstract}
Four new species of Elaphoglossum sect. Elaphoglossum are described: E. amparoanum A. Rojas, E. chocoense A. Rojas, E. fayorum A. Rojas and $E$. luciae A. Rojas. The first species is related to $E$. variolatum Mickel, but differs from it by having rhizome scales bicolorous and long ciliate margin. The second species is different to E. moranii Mickel by having narrower rhizome, shorter stipe, elliptic blade, attenuate blade base, shorter fertile fronds with blackish costa and present at lower altitudinal distribution. After that, the third species differs from E. lingua (C. Presl) Brack. by pale rhizome scales with long ciliate margin, shorter fronds and blade scales denser and lighter. Finally, the fourth species is related to E. atrosquamatum Mickel, but with relative longer fronds and broader blade. The geographical distributions of $E$. guatemalense (Klotzsch) T. Moore, E. longicrure Christ, E. minutum (Pohl ex Fée) T. Moore and E. nicaragüense A. Rojas are expanded.
\end{abstract}

\section{Keywords}

Dryopteridaceae, Elaphoglossum, Ferns, New Records, New Species, Section Elaphoglossum, Taxonomy

\section{Introduction}

Section Elaphoglossum is the largest group of the genus and is characterized by hydathodes absent, phyllopodia present and medium sized blades with stellate blade scales (Mickel \& Atehortúa 1980). Subsection Pachyglossa is characterized by subcoriaceous to coriaceous blade and minute stellate blade scales (vs. chartaceous blade and scales absent in subsection Tenuifolia) [1].

Rouhan et al. performed a phylogenetic analysis of the fern genus Elaphoglossum using two non-coding chloroplast spacers: trnL-trnF and rps4-trnS, ob- 
taining a cladogram based in strict consensus of 1008 most parsimonious combined trees that reveal two well supported subclades in section Elaphoglossum: Pachyglossa and Platyglossa [2], each of which corresponds to a subsection defined by Christ [3]. Also, two species: E. aemulum (Kaulf.) Brack. and E. glaucum T. Moore appeared as different groups to sect. Elaphoglossum and the authors proposed they as new sections. However, no morphological characters were associated for recognize the new sections and subsections in section Elaphoglossum, also the new subsections weren't described formally.

Skog et al. [4] investigated the fern genus Elaphoglossum using cpDNA sequence data from rbcL, trnL-F, and rps4-trnS, and encountered two groups from section Elaphoglossum: Pachyglossa and Platyglossa clades. However the position of E. glaucum within section Elaphoglossum is unresolved by MP and Bayes sequence analyses, the taxon shares insertion rt11 with subsection Pachyglossa, which suggests a closer affinity with this subsection than with subsection Platyglossa. Interestingly, inclusion of E. glaucum in the subsection Pachyglossa cannot be rejected by the nonparametric Templeton [5] test, whereas inclusion in the subsection Platyglossa is rejected by the same test. The authors auditioned morphological characters in the maximum parsimony tree, and defined Pachyglossa clade with leaves less than $30 \mathrm{~cm}$ long and rhizomes narrow, long and creeping, and Platyglossa clade with leaves more than $30 \mathrm{~cm}$ long and rhizomes thick compact, but apparently the trends in morphological characters were associated on the contrary.

Moran et al. [6] studied perispore structure using a scanning electron microscope and compared the spore ornamentation with a previously published phylogenetic analysis [2] of the genus based on two chloroplast noncoding DNA regions, trnL-trnF and rps4-trnS. They analyzed presence and continuously of broad folds or cristae, perispore character, presence of spines and perforations, and discovered three sinapomorphic characters from sect. Pachyglossa: cristate perispore, spines and perforations; however none is constant for separate the two subsections.

Because previous authors do not support the description of the new sections, or not defined appropriately morphologically sections and subsections included in section Elaphoglossum, the author of this paper prefers to maintain the classification system proposed by Mickel \& Atehortúa [7].

\section{Materials and Methods}

The new species here considered are the result of comparisons with specimens of other Neotropical species, and a review of related species and keys from the neotropical ferns by Gómez \& Arbeláez [8], Mickel [9] [10] [11] [12], Mickel \& Beitel [13], Mickel \& Smith [14], and Murillo et al. [15] and other papers in the Elaphoglossum genus by Kessler \& Mickel [16], Mickel [17] [18] [19] [20] [21], Neves \& Salino [22], Rojas [23] [24] [25] [26] [27] and Rojas \& Rodríguez [28]. The examined specimens are deposited in the following herbaria CR, COL, EAP, F, HUA, MEXU, MO, TEFH, UC, US and USJ (acronyms following Thiers, [29]). 
To ensure the correct application names, original type material or digital type images were examined as available (Jstor Global Plants (http://plants.jstor.org/)), and the new names were corroborated with International Plant Name Index (http://www.ipni.org/ipni/plantnamesearchpage.do).

\section{Results}

\subsection{New Species}

\subsubsection{Elaphoglossum amparoanum}

A. Rojas, sp. nov. (Figure 1, Figure 2)

TYPE: COLOMBIA. Antioquia: San Andrés Municipio, km 13 of road Toledo-San José de La Montaña, 06 $54^{\prime} \mathrm{N}, 75^{\circ} 41^{\prime} \mathrm{W}, 2300$ m, 13 May 1988, J. Zarucchi \& J. Betancur 6521 (holotype: MO).

Diagnoses. Ab Elaphoglosso variolato Mickel rhizomatis squamis ciliatas vel stellatis (adversus integris ad costo ciliatis) et nigras (adversus atroflavescentibus) differt.

Description. Epiphytic; rhizomes $2-3 \mathrm{~mm}$ in diameter, creeping, fronds 13 $25 \mathrm{~mm}$ distant; rhizome scales 2 - 3 by $1-1.5 \mathrm{~mm}$, ovate to lanceolate, bicolorous, blackish with the base and margin commonly yellowish-brown, peltate, appressed, marginally long-ciliate; phyllopodia $7-15 \mathrm{~mm}$ long; fronds $10-42 \mathrm{~cm}$

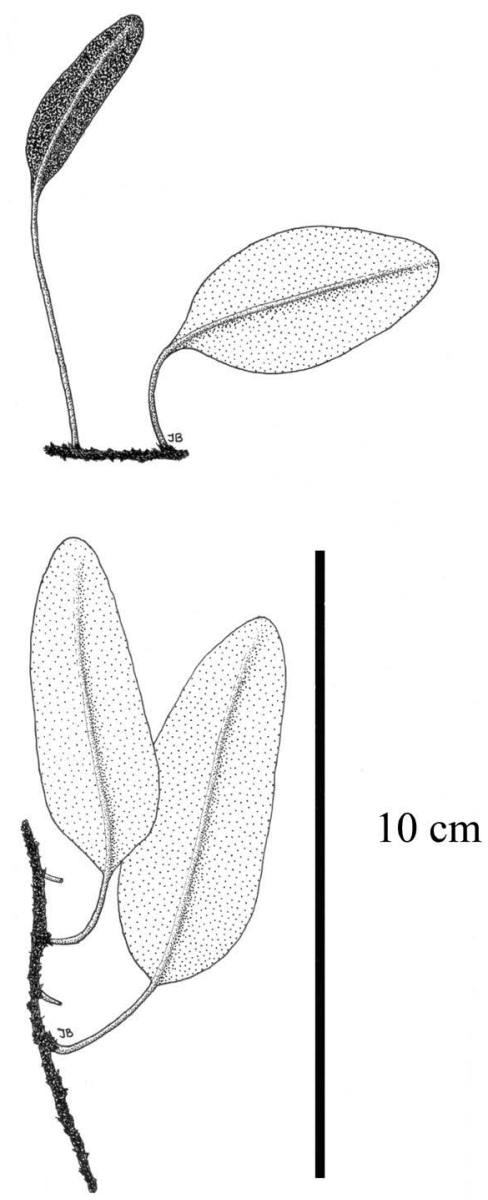

Figure 1. Elaphoglossum amparoanum (J. Zarucchi \& J. Betancur6521, MO). A. Habit. 


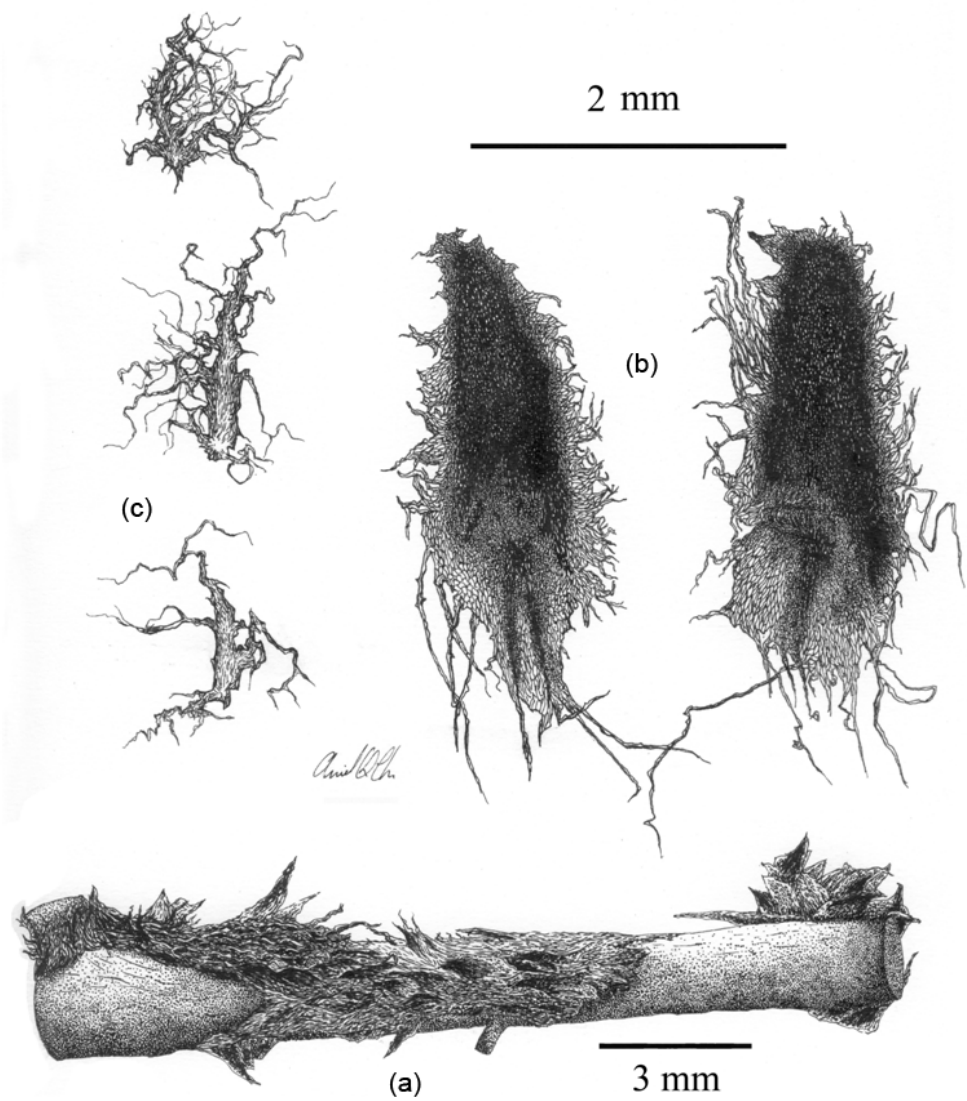

Figure 2. Elaphoglossum amparoanum (J. Zarucchi \& J. Betancur 6521, MO). (a) Rhizome detail; (b) Rhizome scales; (c) Blade scales.

long; stipe 3 - $22 \mathrm{~cm}$ long, brown, scaly, the scales similar to the rhizome scales; blade 7 - 21 by $2.5-7.2 \mathrm{~cm}$, ovate to lanceolate, coriaceous, basally obtuse, apically obtuse; costa brown at base, higher up stramineous, scaly, the scales 1 - 2 by $0.7-1 \mathrm{~mm}$, ovate, gold-brown, medium-dense, marginally with lateral processes; blade abaxially with scales $0.5-1.5 \mathrm{~mm}$ long, rounded to ovate (including cilia), stellate, yellowish-brown; blade scales adaxially similar in size and form to abaxialy scales but whitish and sometimes deciduous; veins little evident; hydathodes absent; fertile fronds $15 \mathrm{~cm}$ long; stipe $9.5 \mathrm{~cm}$ long; blade 5.5 by $1.4 \mathrm{~cm}$, oblong-elliptic, basally broadly cuneate, apically obtuse to rounded; intersporangial scales absent.

Etymology. The new species is dedicated to Luz Amparo Triana Moreno, who works with Colombian ferns.

Distribution. Known only from Colombia in the Cordillera de Los Andes at $2100-2600 \mathrm{~m}$.

Additional revised specimens (paratypes). COLOMBIA. Antioquia: Laguna de Guarne, Dec. 1935 Bro. Daniel s.n. (COL); ibídem., San Pedro, Feb. 1937, Bro. Daniel 1020 (COL, US); Municipio de Belmira, vereda El Yerbal, sitio Los Patos, margen izquierda del Río Chico, alto de Sabanazos, $06^{\circ} 35^{\prime} \mathrm{N}, 75^{\circ} 32^{\prime} \mathrm{W}, 2600 \mathrm{~m}$, 24 Apr. 1991, D. Echeverri et al. 454 (MO). Antioquia-Chocó: at and on both sides of the principal ridge $\mathrm{W}$ of La Mansa, at ca. Km 105.5 of the Ciudad 
Bolívar-Quibdó road, 2100 - 2200 m, 4 Apr. 1971, D. Lellinger \& E. de la Sota 939 (US). Cauca: Parque Nacional Munchique, Km 50 - 55 along road above Uribe, 1875 - 2256 m, 25 Apr. 1979, J. Luteyn et al. 7483 (COL). Cundinamarca: Muni- cipio San Bernardo, Vereda Santa Rita, Hacienda El Placer, 2150 - 2200 m, 27 Jul. 1981, S. Díaz 3211 (COL); San Miguel a Aguabonita, carretera Aguabonita- Fusagasugá, 2000 m, 15 Apr. 1946, J. Duque 3304 (COL). Huila: Municipio San Adolfo, Parque Nacional Cueva de Los Guácharos, 1800 m, 6 - 7 Feb. 1976, Acosta 1041 (COL); Cordillera Oriental, 15 km SE of Garzón, 7200 ft. [=2190 m], 1 Feb 1945, E. Little 9348 (US).

In its creeping rhizome and blade shape, the new species is similar to Elaphoglossum variolatum Mickel; however, E. amparoanum differs by its ciliate to stellate (vs. entire to short-ciliate) and bicolorous rhizome scales with blackish center (vs. concolorous). The new species is also similar to E. lingua (C. Presl) Brack., but differs from it by its ciliate to stellate (vs. entire to short-ciliate) rhizome scales, yellowish-brown (vs. blackish) and medium-dense (vs. sparse to glabrescent) blade scales (Figure 1, Figure 2).

\subsubsection{Elaphoglossum chocoense}

\section{A. Rojas, sp. nov. (Figure 3, Figure 4)}

TYPE: COLOMBIA. Chocó: Mpio. Bahía Solano, corregimiento El Valle, Parque Nacional Natural Ensenada de Utría, serranía del Baudó, camino de Utría a El Valle, $6^{\circ} 06^{\prime} \mathrm{N}, 77^{\circ} 21^{\prime} \mathrm{W}, 30-50 \mathrm{~m}, 27$ Jun 1999, J. Betancour \& $N$. García 8062 (holotype: HUA; isotype: COL).

Diagnoses. Elaphoglossum chocoense a E. moranii Mickel rhizomatibus angustioribus, stipitibus brevioribus, lamina elliptica, basi laminae attenuata, frondibus fertilibus brevioribus costa nigrescenti et altitudinale inferna distributio differt.

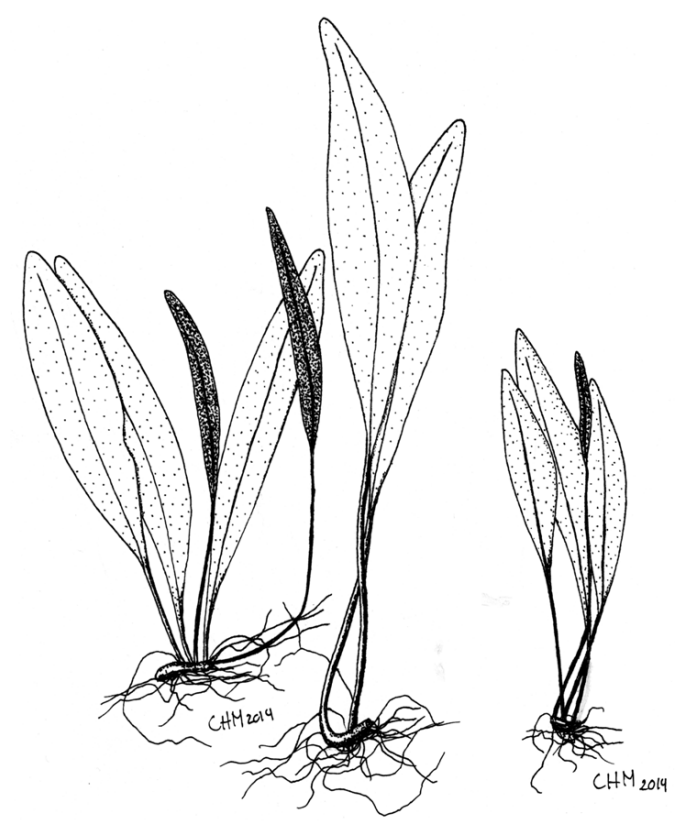

Figure 3. Elaphoglossum chocoense (J. Betancour \& N. García 8062, HUA). A. Habit. 

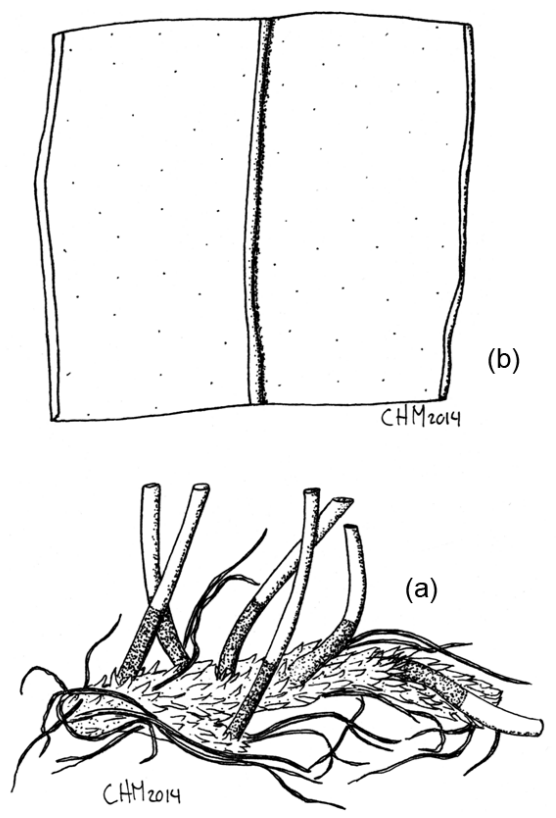

Figure 4. Elaphoglossum chocoense (J. Betancour \& N. García 8062, HUA). A. Rhizome detail. B. Blade detail.

Description. Epiphytic; rhizomes $1-2 \mathrm{~mm}$ in diameter, short-creeping, fronds 2 - $7 \mathrm{~mm}$ distant; rhizome scales 0.7 - 2 by 0.5 - $1 \mathrm{~mm}$, ovate to lanceolate, peltate, appressed, dark brown to blackish, flat, marginally dentate to ciliate with a narrow brown-yellowish ribbon; fronds $11-19 \mathrm{~cm}$ long; stipe $2-3 \mathrm{~cm}$ long, stramineous, scaly at base, the scales $0.5-1.5$ by $0.3-0.7 \mathrm{~mm}$, similar to the rhizome scales but gradually shorter towards the lamina; phyllopodia $7-10 \mathrm{~mm}$ long; blade $8-14$ by $1.4-2.8 \mathrm{~cm}$, elliptic, coriaceous, basally attenuate, apically broadly acute to obtuse; costa stramineous, basally scaly, the scales $0.5-1.0$ by $0.2-0.5 \mathrm{~mm}$ similar to stipe scales but smaller; blade abaxially gabrous, with resinous dots or with minute (less than $0.1 \mathrm{~mm}$ ) stellate and orange scales; blade adaxially glabrous; veins not evident; hydathodes absent; fertile frond 11.5 - 15 cm long; stipe $5.5-8.0 \mathrm{~cm}$ long; blade $6-8$ by $0.7-1.0 \mathrm{~cm}$, narrowly elliptic, base attenuate, apex acute, with blackish costa abaxially; intersporangial scales absent.

Etimology. The specific epithets make referent to type locality.

Distribution. Known only from the type in Colombia in the biogeographic Chocó at $30-50 \mathrm{~m}$.

Elaphoglossum chocoense differs from E. moranii Mickel by having narrower (1 - $2 \mathrm{~mm}$ vs. $2-3 \mathrm{~mm}$ ) rhizome, shorter $(2-3 \mathrm{~cm}$ long vs. $4-18(-26) \mathrm{cm})$ stipe, elliptic (vs. narrowly oblong to linear-lanceolate) blade, attenuate (vs. narrowly cuneate) blade base, shorter $(11.5-15 \mathrm{~cm}$ vs. $17-41(-50) \mathrm{cm})$ fertile fronds with blackish (vs. strawish) costa and present at lower altitudinal distribution (30 - $50 \mathrm{~m}$ vs. $500-2000 \mathrm{~m}$ ). The new species is also similar to E. lingua (C. Presl) Brack., but differs from it by its shorter (2-3 cm long vs. $4-17 \mathrm{~cm}$ ) stipe, elliptic (vs. lanceolate) blade, attenuate (vs. broadly cuneate) blade base, shorter (11.5 - $15 \mathrm{~cm}$ vs. $20-50 \mathrm{~cm}$ ) fertile fronds and present at lower altitudin- 
al distribution (30 - 50 m vs. 900 - 2000 (- 2500) m) (Figure 3, Figure 4).

\subsubsection{Elaphoglossum fayorum}

A. Rojas, sp. nov. (Figure 5, Figure 6)

TYPE: ECUADOR. Pichincha: Quito Cantón, Río Guajalito Reserve, $10 \mathrm{~km} \mathrm{~W}$ of Chiriboga, km 59 on old road Quito-Santo Domingo, $00^{\circ} 14^{\prime} \mathrm{S}, 78^{\circ} 48^{\prime} \mathrm{W}, 1900$ - 2100 m, 16 Jun 1991, A. Fay \& L. Fay 3129 (holotype: MO; isotype: NY).

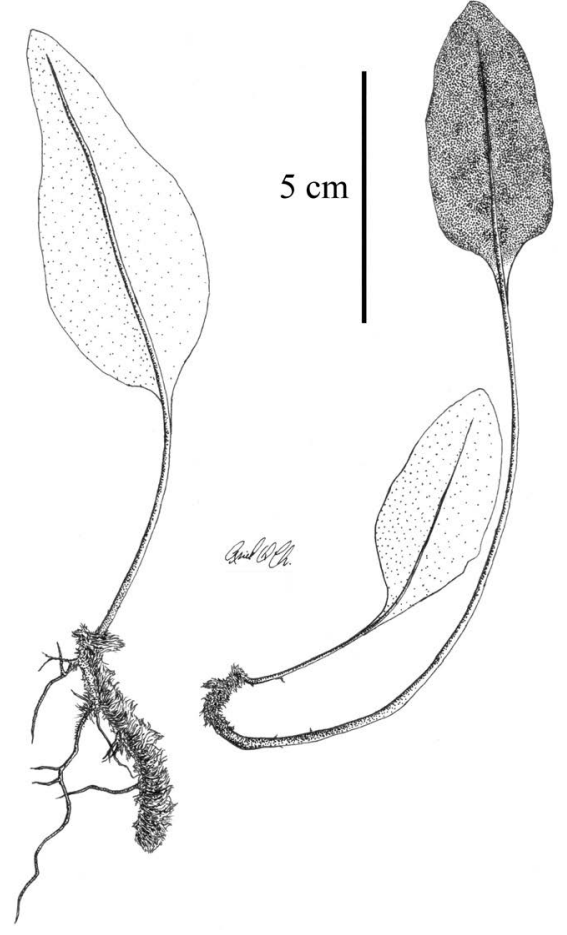

Figure 5. Elaphoglossum fayiorum (A. Fay \& L. Fay3129, MO). A. Habit.

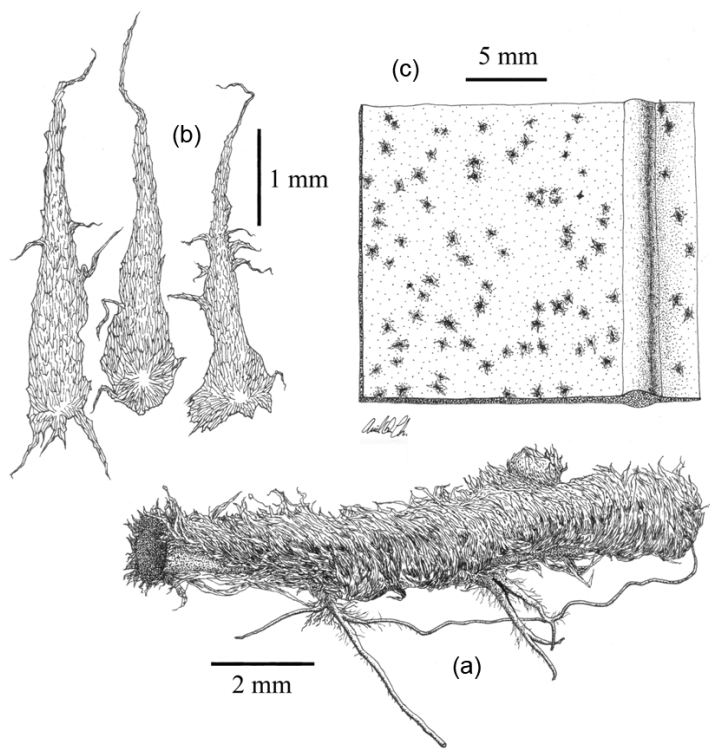

Figure 6. Elaphoglossum fayiorum (A. Fay \& L. Fay 3129, MO). A. Rhizome detail. B. Rhizome scales. C. Blade detail. 
Diagnoses. Ab Elaphoglosso lingua (C. Presl) Brack. rhizomatis squamis densioribus longioribus auratis, lamina breviore, laminae squamis densioribus differt.

Description. Epiphytic; rhizomes $1-2 \mathrm{~mm}$ in diameter, creeping, fronds 5 $15 \mathrm{~mm}$ distant; rhizome scales 2 - 4 by $0.5-1 \mathrm{~mm}$, lanceolate, gold to reddish brown, crisped, marginally with lateral processes dense at the base and sparse elsewhere; fronds 6 - $10 \mathrm{~cm}$ long; stipe $2.5-4 \mathrm{~cm}$ long, stramineous, scaly at base, the scales $1-2$ by $0.3-1 \mathrm{~mm}$, similar to the rhizome scales but gradually shorter towards the lamina; phyllopodia 2 - $9 \mathrm{~mm}$ long; blade 3.5 - 6 by $1.3-2.5$ $\mathrm{cm}$, ovate, coriaceous, basally obtuse, apically broadly acute to obtuse; costa stramineous, scaly, the scales similar to abaxial blade scales; blade abaxially scaly, the scales $0.5-1.5$ by $0.3-0.6 \mathrm{~mm}$, rounded and stellate to lanceolate with long ciliate margin; blade adaxially glabrous; veins few evident; hydathodes absent; fertile frond $14.5 \mathrm{~cm}$ long; stipe $10.3 \mathrm{~cm}$ long; blade 4.2 by $1.8 \mathrm{~cm}$, lanceolate, base obtuse, apex obtuse to round; intersporangial scales absent.

Etimology. The specific epithet honors Alice and Luis Fay, collectors of the type material.

Distribution. Known only from the type in Ecuador in Cordillera de Los Andes at $1900-2100 \mathrm{~m}$.

Elaphoglossum fayorum differs from E. lingua (C. Presl) Brack. by the longer (2- $4 \mathrm{~mm}$ vs. $1-2 \mathrm{~mm}$ ), gold to reddish brown (vs. dark brown to blackish) and basally ciliate (vs. entire to dentate) rhizome scales, smaller ( 3.5 - 6 by $1.3-2.5$ cm vs. 8 - 24 by $2.7-6.5 \mathrm{~cm}$ ) blades and dense (vs. sparse or absent) blade scales. It is also similar to E. pteropus C. Chr.; however, E. fayorum differs by gold to reddish brown (vs. dark brown) and ciliate (vs. entire) rhizome scales (Figure 5 , Figure 6).

\subsubsection{Elaphoglossum luciae}

A. Rojas, sp. nov. (Figure 7, Figure 8)

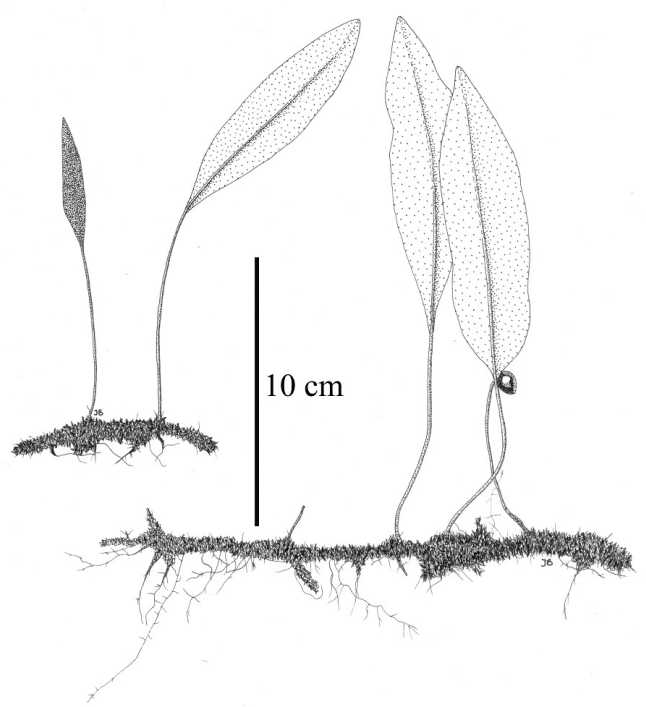

Figure 7. Elaphoglossum luciae (B. Boyle \& S. Chapotin 5965, MO). A. Habit. 


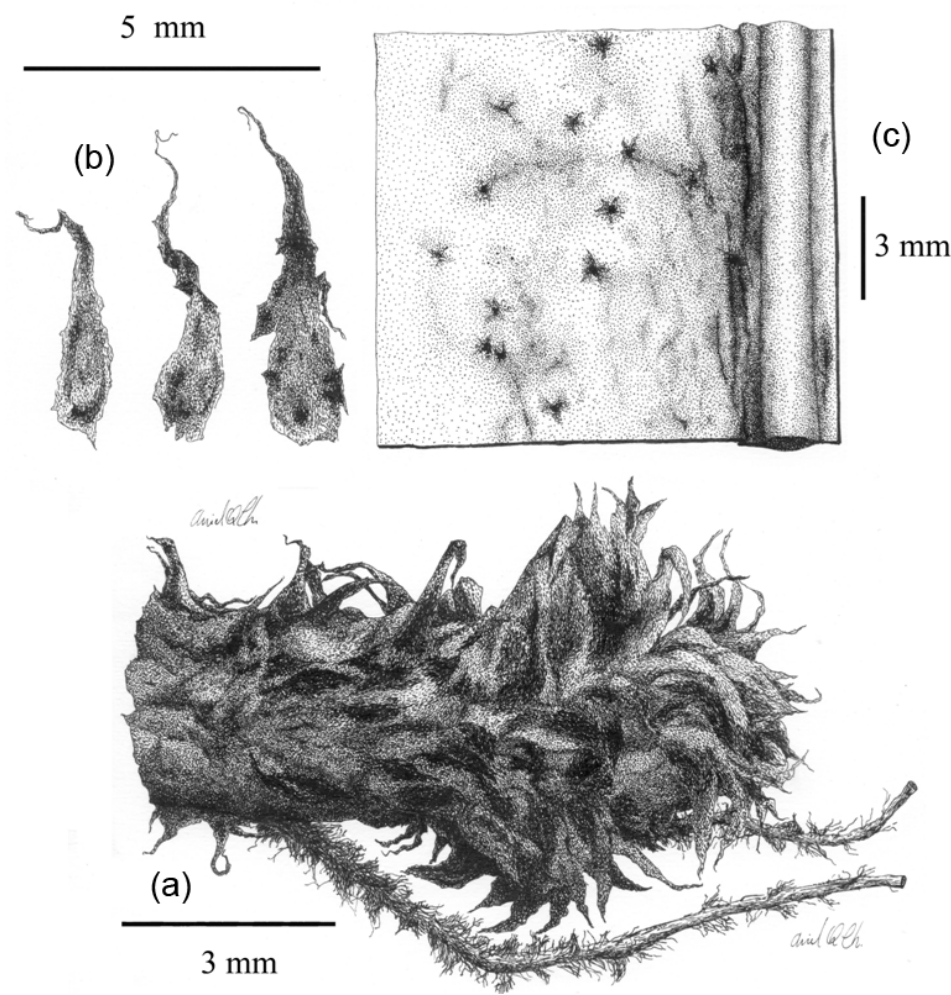

Figure 8. Elaphoglossum luciae (B. Boyle \& S. Chapotin 5965, MO). A. Rhizome detail. B. Rhizome scales. C. Blade detail.

TYPE: COSTA RICA. San José: Pérez Zeledón, Cerro de La Muerte, trail from microwave towers to highway, $09^{\circ} 33^{\prime} 30^{\prime \prime} \mathrm{N}, 83^{\circ} 43^{\prime} 04^{\prime \prime} \mathrm{W}, 3450 \mathrm{~m}, 9 \mathrm{Jul} 2000, B$. Boyle \& S. Chapotin 5965 (holotype: USJ; isotypes: CR, NY).

Diagnoses. Ab Elaphoglosso atrosquamato Mickel rhizomatis crassiore et lamini ovatis ad ovato-lanceolatis (adversus anguste ellipticam) differt.

Description. Terrestrial; rhizomes $2-3 \mathrm{~mm}$ in diameter, moderate to longcreeping, fronds 5 - $15 \mathrm{~mm}$ distant; rhizome scales 5 - 8 by $0.5-15 \mathrm{~mm}$, linear-lanceolate, dark brown to blackish, twisted, patent to curved, marginally entire to long-ciliate near the apex; fronds $7-22 \mathrm{~cm}$ long; phyllopodia $5-10 \mathrm{~mm}$ long; stipe $1 / 3-1 / 2$ of the frond length, scaly, the scales $2-4$ by $0.4-0.8 \mathrm{~mm}$, linear-lanceolate, brown to dark brown, patent, sparse to dense, marginally entire; blade 5 - 9 by $1.5-3 \mathrm{~cm}$, elliptic, coriaceous, basically cuneate, apically acute to acuminate; costa stramineous, scaly, the scales $1-2$ by $0.3-0.7 \mathrm{~mm}$, linearhastate, dark brown to blackish, dense, marginally ciliate, commonly with short lateral extensions, mixed with scales 0.5 - $1 \mathrm{~mm}$ indiameter, stellate to linearhastate, blackish, sparse, marginally ciliate; veins few evident; hydathodes absent; fertile fronds 10 - $18 \mathrm{~cm}$ long; stipe 1/2 - 2/3 of the frond length; fertile blade 4 7 by $1.5-2.0 \mathrm{~cm}$, elliptic, basally cuneate, apically acute; intersporangial scales absent.

Etimology. The new species is dedicated to my daughter, Diana Lucía, because she is an incentive to continue with my work.

Distribution. Known only from Costa Rica in Cordillera de Talamanca at 
(2000-) 2600 - $3400 \mathrm{~m}$.

Additional revised specimens (paratypes). COSTA RICA. Cartago: Cantón de Paraíso, investigation area of CATIE, 09³3'30"N, 8341'30"W, 2600 - $2700 \mathrm{~m}$, 19 December 1990, J. Bittner 142 (CR); Estación Biológica Cerro de La Muerte, (run by Federico Valverde), 9 $33^{\prime} 40^{\prime \prime} \mathrm{N}, 83^{\circ} 44^{\prime} 30^{\prime \prime W}, 3140 \mathrm{~m}, 20$ Jan 2001, $R$. Moran 6333 (USJ); S slopes of Volcán Turrialba, near the Finca del Volcán Turrialba, 2000 - 2400 m, P. Standley 35252 (US); ibídem, P. Standley 35253 (US); ibídem., P. Standley 35310 (US); Volcán Turrialba, 3300 m, A. Torres 24 (US); ibídem., A. Torres 134 (US). Cartago/San José: Cantones de Paraíso/Pérez Zeledón, Carretera Interamericana, entre Cerro Estaquero y la entrada a las torres, 09³ $33^{\prime} 35^{\prime \prime} \mathrm{N}, 83^{\circ} 45^{\prime} 25^{\prime} \mathrm{W}, 3100$ - 3300 m, 24 Apr. 1998, A. Rojas 4522 (INB); road from Cartago to San Isidro del General (Pan American Hwy., Rte. 2), $\mathrm{km} 87-88$, ca. $1 \mathrm{~km} \mathrm{NW}$ of Asunción, 9 $34^{\prime} 48^{\prime \prime} \mathrm{N}, 83^{\circ} 45^{\prime} 36^{\prime \prime} \mathrm{W}, 3200 \mathrm{~m}, 29$ Jan. 1986, A. Smith \& T. Béliz 2021 (CR, MO, UC); Interamerican Hwy., ca. 1.5 km NW of Restaurant La Georgina, 3100 m, 24 Aug. 1975, J. Utley \& K. Utley 2967 (CR). Limón: Cordillera de Talamanca, southwestern foot of Cerro Kámuk, $09^{\circ} 16^{\prime} \mathrm{N}, 83^{\circ} 02^{\prime} 30^{\prime \prime} \mathrm{W}, 3200$ - 3350 m, 24 Mar 1984, G. Davidse et al. 25953 (CR, MO, UC). San José: Pérez Zeledón, Cerro de La Muerte, trail from microwave towers to highway, $9^{\circ} 33^{\prime} 30^{\prime \prime} \mathrm{N}, 83^{\circ} 43^{\prime} 04^{\prime \prime W}, 3450 \mathrm{~m}, 9$ Jul 2000, B. Boyle \& $S$. Chapotin 5965 (USJ); ibidem, 3350 m, B. Boyle \& S. Stefanovic 5817 (USJ); Cerro Chirripó, SW slopes, along trail from Canaán to summit, nr. La Caverna, 9800 - 10,300 ft [=2985 - $3140 \mathrm{~m}]$, A. Evans et al. 88 (US); Cordillera de Talamanca, near Villa Mills, 9³3'N, 8341'W, 2700 m, 11 Jan. 1990, Merz 613 (CR); Dota, Reserva Forestal Los Santos, Cuenca del Savegre, camino a San Gerardo de Dota, desde la entrada hasta $1.5 \mathrm{~km}, 09^{\circ} 32^{\prime} 14^{\prime \prime} \mathrm{N}, 83^{\circ} 49^{\prime} 27^{\prime \prime} \mathrm{W}, 2900$ 3000 m, 18 Aug 2001, A. Rojas 5488 (CR).

Elaphoglossum luciae differs from $E$. atrosquamatum Mickel by having a thicker ( 2 - $3 \mathrm{~mm}$ vs. $1-1.5 \mathrm{~mm}$ ) rhizome and broader $(1.5-3 \mathrm{~cm}$ vs. $0.3-1.3$ $\mathrm{cm}$ ), ovate to ovate-lanceolate (vs. narrowly elliptic) blades and cuneate (vs. long-decurrent) at the base. Probably it is more closely related to E. hoffmannii (Mett. ex Kuhn) H. Christ due to its blackish rhizome scales and blue-green adaxial blade surface, but differs by relatively shorter (7 - $22 \mathrm{~cm}$ long vs. 12 - 36 $\mathrm{cm}$ ) fronds, longer $1 / 3-1 / 2$ of the frond length vs. ca. 1/5) stipes and shorter [5 $9 \mathrm{~cm}$ long vs. $9-35 \mathrm{~cm}$ ] elliptic (vs. narrowly elliptic to linear-elliptic) blades. Also is similar to E. antisanae (Sodiro) C. Chr. but the scales are narrower and dark brown to blackish fully (Figure 7, Figure 8).

\subsection{New Records}

\subsubsection{Elaphoglossum guatemalense}

(Klotzsch) T. Moore, Index Filicum, 357. 1862.

Acrostichum guatemalense Klotzsch, Allgemeine Gartenzeitung 23: 66. 1855. Type: Guatemala (from cult. in Potsdam, Germany, grown from spores of unknown locality in Guatemala), Anon. (B?).

Distribution. Mexico, Guatemala, Belize, El Salvador, Honduras and Nicara- 
gua.

Material of new distribution. NICARAGUA. Matagalpa: Río Tuma, Aug 1957, J. Salas \& B. Taylor 3010 (EAP).

\subsubsection{Elaphoglossum longicrure}

Christ, Bulletin de l'Herbier Boissier, sér. 2, 7, 273. 1907.

Type: Costa Rica: San José, Tablazo, 1900 m, Sep 1906, C. Biolley s.n. (Holotype: P!).

Distribution. Guatemala, El Salvador, Honduras, Nicaragua, Costa Rica, Panama and Colombia.

Material of new distribution. GUATEMALA. Alta Verapaz: road from Chama x Cobán, 3000 ft. [=915 m], H. Johnson 543 (US); vicinity of Cobán, about 1300 m, P. Standley 90853 (F). Isabal: along Río Frío, 50 - 75 m, J. Steyermark 41631 (US). Quezaltenango: high barranco along Río Samalá, between Santa María de Jesús and Calahuaché, 1200 - 1300 m, J. Steyermark 33874 (F). San Marcos: Finca El Porvenir, Loma Trocodoná, S facing slopes of Volcán Tajumulco, 1300 - 1500 m, J. Steyermark 37538 (F, US). Sololá: Volcán Atitlán, 1700 - 3800 m, J. Steyermark 47346 (US); Río Bravo, in vicinity of Finca Mocá, S facing slopes of Volcán Atitlán, 1000 - 1100 m, J. Steyermark 47965 (F, US). Suchitepéquez: Finca Moca, W. Muenscher 12136 (F).

EL SALVADOR. Ahuachapán: El Imposible Reserve, Campana, $13^{\circ} 51^{\prime} \mathrm{N}$, 8954'W, 1400 m, A. Monro et al. 2018 (MEXU).

HONDURAS. El Paraíso: Mpio. San Antonio de Oriente, La Labranza, Montaña de Uyuca, J. Linares \& R. Dressler 3810 (MEXU); Ciudad de Yuscarán, Cerro Monserrat, 2000 m, M. Espinal 120 (CR). Francisco Morazán: W sloep of Cerro de Uyuca, along trail from Las Flores toward Tatumbla, 1500 - 1600 m, 17 Aug 1949, P. Standley 22792 (EAP); Sierra San Juancito, road to San Juancito, J. Swallen 11129 (US). Ocotepeque: El Portillo on Cordillera Merendón, $20 \mathrm{~km}$ from Nueva Ocotepeque, 1800 m, 28 Aug 1968, A. Molina 22335 (EAP, NY).

\subsubsection{Elaphoglossum minutum}

(Pohl ex Fée) T. Moore, Index Filicum, 12. 1857. Acrostichum minutum Pohl ex Fée, Mémoires sur les Familles des Fougères 2: 39, pl. 10. 1844 [1845]. Type: Brazil, near Goyas, J. Pohl s. n. (Holotype: W, n. s.).

Distribution. El Salvador, Costa Rica, Panama, Colombia, Venezuela, Ecuador, Peru, Bolivia, Brazil, Jamaica and La Española.

Material of new distribution. EL SALVADOR. Santa Ana: Áraferrand des Santa Ana, 2380 m, 3 Jun 1953, E. Lotschert 309 (EAP).

\subsubsection{Elaphoglossum nicaragüense}

A. Rojas, Revista de Biología Tropical, 51, 1 - 32. 2003.

Type: Nicaragua, Jinotega, Laguna Miraflores, $13^{\circ} 15^{\prime} \mathrm{N}, 86^{\circ} 15^{\prime} \mathrm{W}, 1250$ - 1300 m, 10 - 11 Jun. 1981, J. Henrich \& W. Stevens 299 (holotype: MO!; isotypes: CR!, MEXU!, NY!, UC!).

Distribution. Honduras and Nicaragua.

Material of new distribution. HONDURAS. Comayagua: Barranco de Trin- 
cheras, $20 \mathrm{~km} \mathrm{~N}$ of Siguatepeque, $1200 \mathrm{~m}, 8$ Apr. 1951, L. Williams \& A. Molina 17605 (EAP). Intibucá: 1600 m, 21 May 1964, A. Molina \& A. Molina 13850 (EAP). Santa Bárbara: 1000 m, 9 Apr. 1951, L. Williams \& A. Molina 17695 (EAP).

\section{Acknowledgements}

I thank Cyril Nelson for the translation of the Latin diagnosis, to the curators of Instituto de Ciencias Naturales-Universidad Nacional de Colombia (COL), Museo Nacional de Costa Rica herbarium (CR), Escuela Agrícola Panamericana herbarium (EAP), Universidad de Antioquia Herbarium (HUA), Missouri Botanical Garden (MO), New York Botanical Garden (NY), Universidad Nacional Autónoma de Honduras Herbarium (TEFH), University of California Herbarium (UC), National Museum of Natural History Herbarium (US) and Universidad de Costa Rica Herbarium (USJ) for specimen loans, to Ariel Quesada, Jenny Bermúdez and Christian Herrera for their excellent illustrations, and to the anonymous reviewers for their helpful suggestions.

\section{References}

[1] Rojas, A.F. (2003) New Taxa in the Elaphoglossum Sect. Elaphoglossum Subsect. Pachyglossa (Lomariopsidaceae) from Mexico and Central America. Revista de Biología Tropical, 51, 1-32.

[2] Rouhan, G., Dubuisson, J.Y., Rakotondrainibe, F., Motley, T.J., Mickel, J.T., Labat, J.N. and Moran, R.C. (2004) Molecular Phylogeny of the Fern Genus Elaphoglossum (Elaphoglossaceae) Based on Chloroplast Non-Coding DNA Sequences: Contributions of Species from the Indian Ocean Area. Molecular Phylogenetics and Evolution, 33, 745-763.

[3] Christ, K.H. (1899) Monographie des Genus Elaphoglossum, Neue Denkschr. Allg. Schweiz. Ges. Gesammten Naturwiss. Edition. Neue Denkschriften der Allgemeinen Schweizerischen Gesellschaft für die Gesammten Naturwissenschaften Edition.

[4] Skog, J.E., Mickel, J.T., Moran, R.C., Volovsek, M. and Zimmer, E.A. (2004) Molecular Studies of Representative Species in the Fern Genus Elaphoglossum (Dryopteridaceae) Based on cpDNA Sequences rbcL, trnL-F, and rps4-trnS. International Journal of Plant Sciences, 165, 1063-1075.

http://www.jstor.org/stable/10.1086/423877

https://doi.org/10.1086/423877

[5] Templeton, A.R. (1983) Convergent Evolution and Non-Parametric Inferences from Restriction Fragment and DNA Sequence Data. In: Weir, B., Ed., Statistical Analysis of DNA Sequence Data, Marcel Dekker, New York, 151-179.

[6] Moran, R.C., Garrison, J. and Rouhan, G. (2007) Spore Morphology in Relation to Phylogeny in the Fern Genus Elaphoglossum (Dryopteridaceae). International Journal of Plant Sciences, 168, 905-929.

http://www.journals.uchicago.edu/doi/10.1086/518269 https://doi.org/10.1086/518269

[7] Mickel, J.T. and Atehortúa, L. (1980) Subdivision of the Genus Elaphoglossum. American Fern Journal, 70, 47-68. https://doi.org/10.2307/1546218

[8] Gómez, L.D. and Arbeláez, A.L. (2009) Flora de Nicaragua, Helechos (Tomo IV). In: Stevens, W.D., Montiel, O.M. and Pool, A., Eds., Monographs in Systematic Bo- 
tany from the Missouri Botanical Garden, The Missouri Botanical Garden Press, St. Louis, Vol. 116, 64-75.

[9] Mickel, J.T. (1981) Elaphoglossum. In: Smith, A.R. and Breedlove, D.E., Eds., Flora of Chiapas, Part 2, Pteridophytes, California Academy of Sciences, San Francisco, 104-116.

[10] Mickel, J.T. (1991) Elaphoglossum. In: Tryon, R.M. and Stolze, R.G., Eds., Pteridophyta of Peru, Part 4, 17, Dryopteridaceae, Fieldiana Botany, New Series 27, 110-166.

[11] Mickel, J.T. (1992) Elaphoglossum. In: McVaugh, R., Ed., Flora Novo-Galiciana, Vol. 17, Gymnosperms and Pteridophytes, Michigan, 265-281.

[12] Mickel, J.T. (1995) Elaphoglossum. In: Moran, R.C. and Riba, R., Eds., Flora Mesoamericana, Vol. 1, Psilotaceae a Salviniaceae, Universidad Nacional Autónoma de México, Ciudad de México, Mexico, 250-283. http://www.mobot.org/mobot/tropicos/meso/homep_sp.htm

[13] Mickel, J.T. and Beitel, J.M. (1988) Pteridophyte Flora of Oaxaca, México. Memoirs of the New York Botanical Garden, 46, 168-186 and 526-533.

[14] Mickel, J.T. and Smith, A.R. (2004) The Pteridophytes of Mexico. Memoirs of the New York Botanical Garden, 88, 282-315 and 826-843.

[15] Murillo, M.T., Murillo, J. and León, A. (2008) Los Pteridófitos de Colombia. Arfo, Bogotá, $533 \mathrm{p}$.

[16] Kessler, M. and Mickel, J.T. (2006) Nineteen New Species of Elaphoglossum (Elaphoglossaceae, Pteridophyta) from Bolivia. Brittonia, 58, 93-118.

[17] Mickel, J.T. (1980) Nine New Elaphoglossums (Elaphoglossaceae) from Mexico. Brittonia, 32, 334-339. https://doi.org/10.2307/2806728

[18] Mickel, J.T. (1987) New Species of Elaphoglossum (Elaphoglossaceae) from Northern South America. Brittonia, 39, 313-339. https://doi.org/10.2307/2807127

[19] Mickel, J.T. (1990) Three New Species of Elaphoglossum from Peru. American Fern Journal, 80, 110-112. https://doi.org/10.2307/1547176

[20] Mickel, J.T. (1992a) New Species of the Fern Genus Elaphoglossum from Mesoamerica. Novon, 2, 368-382. https://doi.org/10.2307/3391496

[21] Mickel, J.T. (1993) Six New Species of Elaphoglossum (Elaphoglossaceae). Brittonia, 45, 213-218. https://doi.org/10.2307/2807103

[22] Neves, L.C. and Salino, A. (2011) New Species of Elaphoglossum Schott ex J.Sm. (Dryopteridaceae) from Brazil. American Fern Journal, 101, 261-264. https://doi.org/10.1640/0002-8444-101.4.261

[23] Rojas, A.F. (1996) Twelve New Species of Elaphoglossum (Elaphoglossaceae) from Costa Rica and Panama. Brenesia, 45-46, 7-26.

[24] Rojas, A.F. (1997) Fourteen New Species of Elaphoglossum (Elaphoglossaceae) from Mesoamerica. Brenesia, 47-48, 1-16.

[25] Rojas, A.F. (2002) New Species, New Combinations and New Distributions in Neotropical Species of Elaphoglossum (Lomariopsidaceae). Revista de Biología Tropical, 50, 969-1006.

[26] Rojas, A.F. (2005) Una nueva especie de Elaphoglossum sección Elaphoglossum (Lomariopsidaceae) en Costa Rica. Lankesteriana, 5, 185-189.

[27] Rojas, A.F. (2010) A New Species in Elaphoglossum Sect. Elaphoglossum Subsect. Pachyglossa (Dryopteridaceae) from Costa Rica and Panama. Novon, 20, 334-337. https://doi.org/10.3417/2008122 
[28] Rojas, A.F. and Rodríguez, W.D. (2012) Nuevos registros de Elaphoglossum (Dryopteridaceae) para el Neotrópico. Actualidades Biológicas, 34, 171-175.

[29] Thiers, B. (2016) Index Herbariorum: A Global Directory of Public Herbaria and Associated Staff. New York Botanical Garden's Virtual Herbarium.

http://sweetgum.nybg.org/science/ih/

\section{Scientific Research Publishing}

Submit or recommend next manuscript to SCIRP and we will provide best service for you:

Accepting pre-submission inquiries through Email, Facebook, LinkedIn, Twitter, etc. A wide selection of journals (inclusive of 9 subjects, more than 200 journals)

Providing 24-hour high-quality service

User-friendly online submission system

Fair and swift peer-review system

Efficient typesetting and proofreading procedure

Display of the result of downloads and visits, as well as the number of cited articles

Maximum dissemination of your research work

Submit your manuscript at: http://papersubmission.scirp.org/

Or contact ajps@scirp.org 